\title{
Conflagration and logging detection
}

\author{
Gokilapriya.P ${ }^{1}$, Varatharaj. $\mathbf{M}^{2}$, Banumathi.P $\mathbf{P}^{3}$, Pavithra.S.B ${ }^{4}$, \\ Gayathiri. $M^{5}$ and Ravivarma. $P^{6}$
}

\author{
Assistant Professor ${ }^{1}$, Department of Electronics and Communication Engineering, Kathir \\ college of Engineering, Tamilnadu, INDIA.
}
Associate Professor ${ }^{2}$, Department of Electronics and Communication Engineering, Kathir college of Engineering, Tamilnadu, INDIA.
Professor $^{3}$, Department of Computer Science and Engineering, Kathir college of Engineering, Tamilnadu, INDIA.

\author{
Bachelor of Engineering Students ${ }^{45}$, Department of Electronics and Communication \\ Engineering, Kathir college of Engineering, Tamilnadu, INDIA.
}

\begin{abstract}
The forest fire and deforestation has an adverse effect in environment. A wild land fire is an uncontrollable fire that occurs mainly in forest areas, it also occurs in urban or agricultural areas. Among the most causes of wildfires, human factors, either intentional or accidental, are the foremost usual ones. The number and impact of forest dangerous fires are expected to grow as a consequence of the worldwide warming. In order to fight against these disasters, it's necessary to adopt a comprehensive and multiple approaches that permit endless situational awareness and instant responsiveness. The main intention of this project is to provide forest security by preventing unauthorized cutting of trees and forest fires. The detection device used to detect the forest fire and deforestation comprises of multiple sensors such as LM35 temperature sensor, MQ3 smoke sensor, Flame sensor and PIR sensor to indicate the fire in different factors. Arduino board and Wemos D1 are used to control all these sensors. The program will be loaded and uploaded in Wemos. An application was developed to connect and work in a single server. The detected information will be send to user mobile and mobile apps using SMS and WiFi interface by the Arduino.

Keywords Arduino UNO, Wemos D1, GSM.
\end{abstract}

\section{Introduction}

Nowadays forest fire occurs very frequently. It causes great damage to the great extension of forest land and destroys habitats like animals and birds. Due to the forest fire some rare species of trees and medicinal plants get extinct. Forest fire increases carbon-di-oxide levels in atmosphere contributing to greenhouse effect and climate changes. It destroys much of the soil and erodes the soil causing flood and landslide. The entire forest ecosystem will also be collapsed. In earlier days fire occurs naturally, ignited by heat from the sun. One of the main reasons of natural occurrence of forest fire is due to the departure of the lightening strike, sometimes collision of two siliceous rocks that also produces spark which results in the minimal impact of fire. Naturally occurring fires can be quickly detected as they have only one outbreak. However most of the wildfires are due to human's uncontrollable activity and carelessness such as lit cigar, not burning debris properly, fireworks, arson and camp fire and also due to the damage of power lines or military accidents. Next to the forest fire deforestation has an adverse effect on society. Development of urbanization and the construction of industries and factories in rural areas are the major reasons which results in deforestation. Since the dams are constructed across the river it results in destruction of forest cover. Farming, cattling, logging for materials also results in deforestation. $70 \%$ of animals and plants live in forest and deforestation leads to destroy their homes, since they provide shelter for some rare species of plants. Deforestation not only reduces the level of ground water but also that results in water scarcity. Logging of trees results in the release of large amount of green house gases into the atmosphere and also leads to global warming. It decreases the level of pollution that could be controlled by the nature warming and climate changes. Soil erosion is being caused by wide spread deforestation. The proposed unique solution helps to overcome the above 
problems by fixing detection sensors using wemos arduino.

\section{Literature Survey}

In the period of 1999, Satellite images was gathered by two main satellites launched for fire detection purposes, the advanced very high resolution radiometer (AVHRR) [4], launched in 1998, and the moderate resolution imaging spectro radiometer (MODIS), launched in 1999, have been used [5,6]. Unfortunately, these satellites can provide images of the regions of the world every two days which may be a while for fire scanning; besides the standard of satellite images can be affected by weather conditions [7]. EYEfi SPARC produced optical sensors, for forest fire detection. EYEfi doesn't provide automatic detection of smoke but plans to introduce it sometime within the future. Simply, whenever the operator notices smoke the EYEfi can provide images for fire agencies. A weather station and lightening detector are included in the system for more accuracy [8, 9]. With the reference [3] this paper was developed. This paper contains numerous motivating factors for the use of an image processing based method of fire detection. The first factor is the rapid development of digital camera technology and CCD or CMOS digital camera. The second factor is that a digital camera can cover large areas with excellent results. The third factor is that the response time of image processing models are better. It proposes an efficient fire detection method using image processing techniques including movement containing region detection supported background subtraction and color segmentation. The algorithm uses $\mathrm{YCbCr}$ color which is better at separating luminance from chrominance. It is used in automatic forest fire alarm system.

\section{Proposed Method}

In older methods they adopted satellite based systems and optical sensors to detect the fire accidents. Recently image processing techniques were developed based on background subtraction color segmentation. The satellite won't be equipped with transponders, antennas, amplification reception, regeneration and downlink transmission fitted to detection of forest fires. Any existing satellite-based observations for forest fires suffer from severe limitations leading to a failure in speedy and effective control for forest areas. The detection device configured to prevent forest fire and deforestation comprises one Arduino board, one Wemos D1, GSM and multiple sensors such as Flame sensor, LM35 Temperature Sensor, MQ3 Smoke Sensor, and PIR Sensor.

Multiple sensors have been used to indicate the fire in multiple factors. To give the SMS notification GSM board has been used. Wemos D1 and Arduino have been connected in serial interface. Since there is no in-built Wi-Fi in Arduino, we have to connect the GSM to Arduino and Arduino to Wemos. All these devices are connected to serial interfaces. As the GSM needs additional power supply from $9 \mathrm{~V}$ to $12 \mathrm{~V}$ hence we have used power adopter. The power is fed parallely to both Arduino and Wemos. The program required for this configuration have been loaded and uploaded in wemos. For testing, mobile hotspot has been used. After uploading the program in wemos d1, Wi-Fi interface tries to connect with mobile hotspot.

Once the connection is established the web interface of the wemos $\mathrm{d} 1$ is viewed in mobile browser using the corresponding IP address shown in the serial monitor and to validate the current status we have to use the IP address shown in serial monitor in the mobile browser in which we have connected the hotspot. To work all these devices a server is needed so a server is created in the mobile app which has been developed for this project. The server connection is initiated from the dedicated mobile app by clicking the server connection button. The wemos start sensing the data and sensing report is displayed in the serial interface and the same will be displayed in the mobile interface.

The fetched data is sent to the user mobile by the WEMOS, using the SMS and Wi-Fi interface. The detected value of flame, smoke concentration, temperature concentration and human movements will be received in SMS. The SMS can be filtered and sent to other user mobiles which are already fed in the program.

\section{Block diagram}

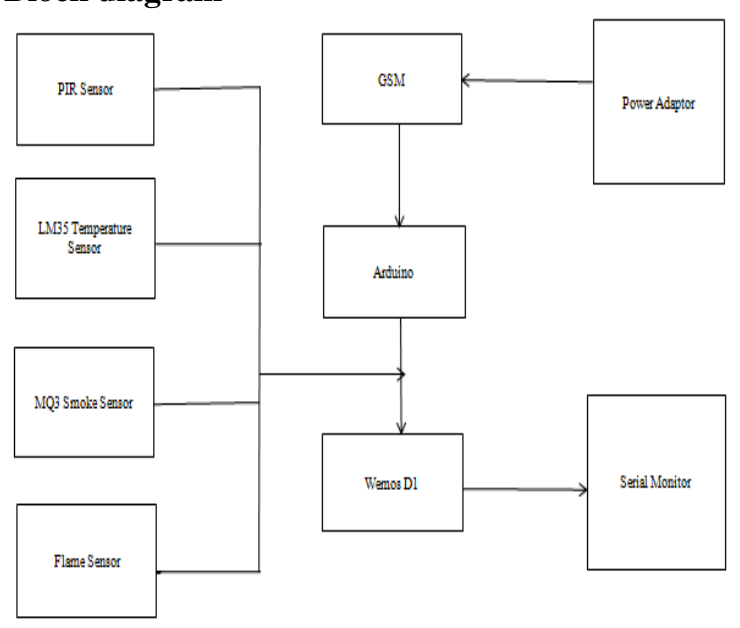

Fig. 1. Block diagram of fire detection system

Figure.1: shows the block diagram of the conflagration and logging detection system. In this the Arduino and Wemos D1 are connected in serial interface. The GSM has been connected to arduino, since ESP in wemos d1 cannot be connected to GSM directly. Hence GSM has been connected to arduino 
and arduino to wemos d1.As GSM needs additional power supply, the GSM has been connected to power adaptor.

The four sensors (PIR sensor, LM35 temperature sensor, MQ3 smoke sensor and flame sensor) are connected to arduino and wemos $\mathrm{d} 1$. The wemos $\mathrm{d} 1$ is connected to serial monitor. The detected information will be send to user mobiles and mobile apps by the wemos d1 by using SMS and Wi-Fi interface.

\section{Flow chart}

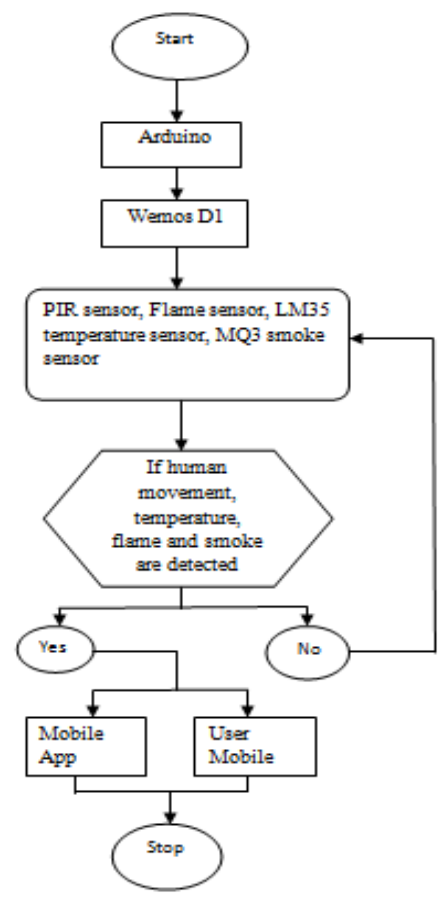

Fig. 2. Flowchart of the detection process

Figure. 2 represents the flowchart of conflagration and logging detection process. If the smoke, flame, temperature and human movements are detected, the detected will be sent to the user mobiles and mobile apps or else the sensors keep detecting the changes in the surroundings.

\section{Hardware Description Arduino and Wemos D1}

Arduino and Wemos D1 are used to control the sensors. The main intension to use Wemos D1 is that it contains many digital $\mathrm{I} / \mathrm{O}$ pins and it is a Wi-Fi module. Its function is similar to that of NODEMCU. The required program for this detection technique is uploaded in Wemos D1. The detected information will be sent by the Wemos D1 with the help of GSM module.

GSM

GSM stands for Global System for Mobile Communication. Mobile voice and data services are transmitted using this digital cellular technology. For transmitting signals GSM makes use of narrowband Time Division Multiple Access (TDMA) technique. In this project it is used to send the message alerts to the user mobiles and mobile apps.

\section{PIR Sensor}

PIR stands for passive infrared sensor. The infrared light (IR) is measured by this electronic device. This sensor detects emitted infrared energy from objects such as humans and animals and also from cars within the sort of heat. The detector itself doesn't emit any energy but passively receives it, detects infrared from the environment.

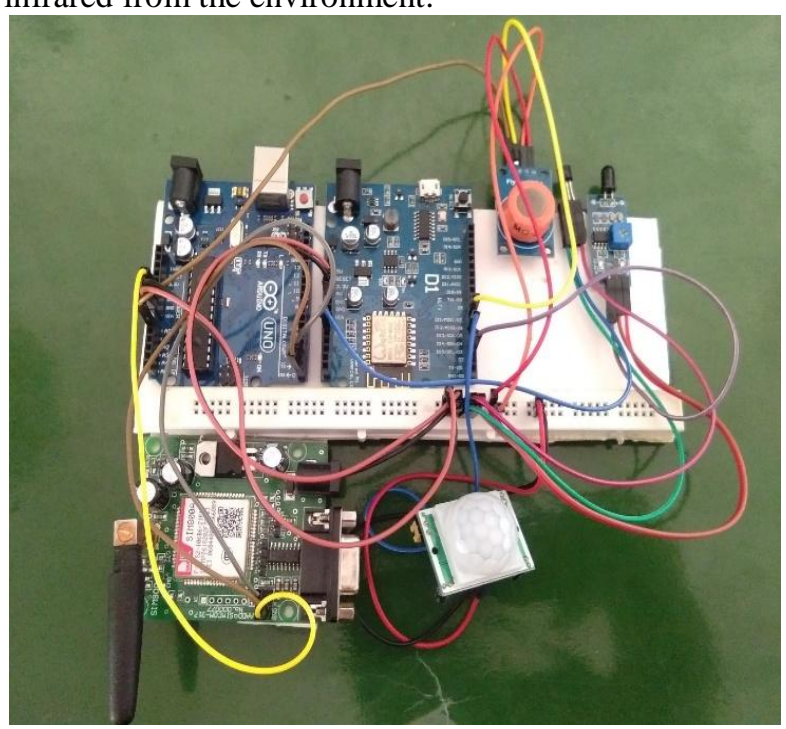

Fig. 3 Hardware of conflagration and logging detection device

\section{LM35 temperature sensor}

Temperature Sensors is used to measure the amount of warmth energy or maybe coldness that's generated within the environment, allowing us to sense or detect any phase change thereto temperature producing either an analog or digital output. Its measurement is accurate and better when compared with a thermistor. This sensor generates a high output voltage than thermocouples and should not need that the output voltage is amplified. The LM35 has an output voltage that's proportional to the Celsius temperature. The scale factor is $01 \mathrm{~V} /{ }^{\circ} \mathrm{C}$.

\section{Flame Sensor}

The flame sensor can detect flame and infrared sources with wavelengths starting from $760 \mathrm{~nm}$ to $1100 \mathrm{~nm}$. The use of LM393 comparator chip provides a clean, stable digital output and driving ability of $15 \mathrm{~mA}$.

\section{Result and Discussion}

The server connection is established in the mobile app created for this project, the wemos start sensing the data and the sensing report is displayed in the serial interface and the same will be displayed in the mobile interface. The fetched data is sent to the user mobile by the wemos, using the SMS and Wi-Fi interface. The SMS can be filtered and can be sent to 
other user mobiles which will be already fed in the program.

\section{Conclusion}

Thus whenever fire accident occurs or an intruder enters into the forest area the sensors will detect and indicate an alert message to the respected user mobiles and mobile apps. We will receive the detected values of human movement, flame, smoke concentration and temperature concentration in SMS.

\section{Future Scope}

This conflagration and logging detection technique will be effective in detecting the fire accidents and gives alert messages to the user mobiles and to the mobile apps in a quick manner. Thus the fire accidents can be avoided and the rare species of trees and animals can be saved.

\section{References}

[1] Antonio Molina-Pico, David Cuesta-Frau, Alvaro Araujo, Javier Alejandre, and Alba Rozas, "Forest Monitoring and Wild Land Early Fire Detection by a Hierarchical Wireless Sensor Network", Journal of Sensors Volume 2016, ArticleID 8325845.

[2] Ahmad A. A. Alkhatib, "A Review on Forest Fire Detection Techniques", International Journal of
Distributed Sensor Networks Volume 2014, Article ID 597368.

[3] Mubarak A. I. Mahmoud and HongeRen, "Forest Fire Detection Using a Rule-Based Image Processing Algorithm and Temporal Variation, Mathematical Problems in Engineering", Volume 2018, Article ID 7612487.

[4] NOAA satellite and information service, "Advanced Very High Resolution RadiometerAVHRR",2012.

[5] NASA, "MOIDS, National Aeronautics and space Administration", 1999, [6] K. Nakau, M. Fukuda, K. Kushida, H. Hayasaka, K. Kimura, and H. Tani, "Forest Fire Detection Based on MODIS Satellite Imagery, and Comparison of NOAA Satellite Imagery with Firefighters Information", 2006.

[7] Y. Aslan, A framework for the use of wireless sensor networks in the forest fire detection and monitoring [M.S.thesis], Department of computer Engineering, The Institute of Engineering and Science Bilkent University, 2010 Review Article A Review on Forest Fire Detection Techniques Ahmad A. A. Alkhatib .

[8] EUFOFINET Project Detection Synthesis of Good Practices, National Forest Centre, Zvolen, Slovakia.

[9 ] S.Mathews, P. Ellis, and J. H. Hurle, Evaluation of Three Systems Bushfire Cooperative Research Centre, Australia, 2010. 